\title{
Nonlocal analysis of the excitation of the geodesic acoustic mode by drift waves
}

Guzdar, P.N.; Kleva, R.G.; Chakrabarti, N.; Naulin, Volker; Juul Rasmussen, Jens; Kaw, P.K.; Singh, R.

Published in:

Physics of Plasmas

Link to article, DOI:

$10.1063 / 1.3143125$

Publication date:

2009

Document Version

Publisher's PDF, also known as Version of record

Link back to DTU Orbit

Citation (APA):

Guzdar, P. N., Kleva, R. G., Chakrabarti, N., Naulin, V., Juul Rasmussen, J., Kaw, P. K., \& Singh, R. (2009).

Nonlocal analysis of the excitation of the geodesic acoustic mode by drift waves. Physics of Plasmas, 16(5), 052514. https://doi.org/10.1063/1.3143125

\section{General rights}

Copyright and moral rights for the publications made accessible in the public portal are retained by the authors and/or other copyright owners and it is a condition of accessing publications that users recognise and abide by the legal requirements associated with these rights.

- Users may download and print one copy of any publication from the public portal for the purpose of private study or research.

- You may not further distribute the material or use it for any profit-making activity or commercial gain

- You may freely distribute the URL identifying the publication in the public portal 


\title{
Nonlocal analysis of the excitation of the geodesic acoustic mode by drift waves
}

\author{
P. N. Guzdar, ${ }_{4}^{1}$ R. G. Kleva, ${ }^{1}$ N. Chakrabarti, ${ }^{2}$ V. Naulin, ${ }^{3}$ J. J. Rasmussen, ${ }^{3}$ P. K. Kaw, ${ }^{4}$ \\ and R. Singh ${ }^{4}$ \\ ${ }^{1}$ Institute for Research in Electronics and Applied Physics, University of Maryland, College Park, \\ Maryland 20742, USA \\ ${ }^{2}$ Saha Institute of Nuclear Physics, 1/AF Bidhannagar, Kolkata 700064, India \\ ${ }^{3}$ Association EURATOM-Ris $\phi$ DTU, Ris $\phi$ National Laboratory for Sustainable Energy, \\ Technical University of Denmark, P.O. Box 49, DK-4000 Roskilde, Denmark \\ ${ }^{4}$ Institute for Plasma Research, Bhat, Gandhinagar 384424, India
}

(Received 23 March 2009; accepted 30 April 2009; published online 28 May 2009)

The geodesic acoustic modes (GAMs) are typically observed in the edge region of toroidal plasmas. Drift waves have been identified as a possible cause of excitation of GAMs by a resonant three wave parametric process. A nonlocal theory of excitation of these modes in inhomogeneous plasmas typical of the edge region of tokamaks is presented in this paper. The continuum GAM modes with coupling to the drift waves can create discrete "global" unstable eigenmodes localized in the edge "pedestal" region of the plasma. Multiple resonantly driven unstable radial eigenmodes can coexist on the edge pedestal. (C) 2009 American Institute of Physics. [DOI: 10.1063/1.3143125]

\section{INTRODUCTION}

Geodesic acoustic modes (GAMs) are low frequency toroidal modes which are primarily electrostatic modes that are observed in a variety of tokamaks. Fluctuations near the GAM frequency are observed in the edge region of tokamaks by a variety of diagnostic methods and these are believed to be excited by nonlinear processes. ${ }^{1-7}$ These modes were first predicted theoretically by Winsor et $a l .{ }^{8}$ Numerous simulations of edge plasmas $^{9-13}$ provided the initial impetus to the experimental investigations. Observations with Doppler reflectometry ${ }^{14,15}$ and multipin probes ${ }^{15,16}$ have identified details of spatial structures and spectral characteristics of the GAMs and have stimulated further theoretical and computational investigations to understand the primary excitation mechanism of these modes. In various experimental studies involving different techniques for the measurement of GAMs, the radial wavenumber $q_{r}$ varies from $0.02<q_{r} \rho_{s}$ $<1$, where $\rho_{s}$ is the ion Larmor radius using the electron temperature. Bicoherence studies ${ }^{15,16}$ in the edge region indicate that a very broad spectrum of the "high" frequency modes $(30 \mathrm{kHz}<f<200 \mathrm{kHz})$ interacts coherently with nearly "monochromatic" GAMs having at most a frequency spread of $20 \%-30 \%$. A recent study ${ }^{14}$ has provided detailed radial structure of the GAMS in the edge region. The key features of these studies display a rather narrow radial extent $(\sim \mathrm{cm})$ of these modes and existence of multiple such eigenmodes whose frequency scales as the GAM frequency. Furthermore these eigenmodes are localized to the edge region of the plasma.

On the theoretical front, it has been shown that GAMs can be excited by three-wave parametric processes involving drift and ion temperature gradient modes. ${ }^{17-21}$ In this paper we present the nonlocal radial eigenstructure of these modes by extending the earlier local parametric interaction studies to include inhomogeneity effects present in the edge region of tokamak plasmas. Typically these modes are continuum modes. It is shown that discrete eigenmodes can be formed in the presence of the nonlinear drive from drift waves. It is shown that there is indeed a multiplicity of such unstable radial eigenmodes and that the frequency of these discrete modes is determined by the resonant wave-matching conditions of the GAM with the primary drift wave and sideband drift wave.

\section{BASIC NONLOCAL EIGENMODE EQUATIONS}

The basic equations used in this investigation are a generalization of the equations used in earlier studies ${ }^{22}$ to account for the background variations of the equilibrium electron density and temperature in a low $\beta$ tokamak plasma. Thus the nonlinear equations used in this study are

$$
\begin{aligned}
& \frac{d n}{d t}-2 \frac{\rho_{s 0} c_{s 0}}{R} n \hat{C} \phi_{e}-\rho_{s 0}^{2} \nabla_{\perp} \cdot\left(n \frac{d}{d t} \nabla_{\perp} \phi\right)=0, \\
& \rho_{s 0}^{2} \nabla_{\perp} \cdot\left(n \frac{d}{d t} \nabla_{\perp} \phi\right)+2 \frac{\rho_{s 0} c_{s 0}}{R} \hat{C} p_{e}=0 .
\end{aligned}
$$

In these equations, $x$ is the radial direction; $\theta$ is the poloidal direction and $\zeta$ the toroidal direction. The density, scalar potential, and temperature are normalized as follows:

$$
\begin{aligned}
& n=\frac{n}{n_{0}(0)}, \quad \phi=\frac{e \phi}{T_{e}(0)}, \quad T_{e}=\frac{T_{e}}{T_{e}(0)}, \quad p_{e}=n T_{e}, \\
& c_{s 0}^{2}=T_{e}(0) / m_{i}, \quad \Omega_{i}=e B_{0} / m_{i} c, \quad \rho_{s 0}=c_{s 0} / \Omega_{i}, \\
& \frac{d}{d t}=\frac{\partial}{\partial t}-\rho_{s 0} c_{s 0} \nabla \phi \times \hat{e}_{\zeta} \cdot \nabla
\end{aligned}
$$

and 


$$
\hat{C}=\frac{\cos \theta}{a} \frac{\partial}{\partial \theta}+\sin \theta \frac{\partial}{\partial x} .
$$

Here $e$ is the electronic charge, $T_{e}(0)$ is the equilibrium electron temperature at $x=0$, the location is the position of the steepest gradient for the equilibrium density and temperature, $a$ the minor radius, $m_{i}$ the mass of the ions, $c_{s 0}$ the ion acoustic speed evaluated for the temperature at $x=0$, and $\Omega_{i}$ the ion cyclotron frequency. $\hat{C}$ is the curvature operator, which includes both the normal curvature contribution (first term in $\hat{C}$ ) and the geodesic curvature. The parallel dynamics for the ions is neglected in this study since for GAMs it makes a minor contribution to the frequency in the edge region (where the safety factor $q$ is large). These equations are valid for large aspect ratio tokamak plasmas with circular flux surfaces.

We investigate the linear parametric excitation of GAMs by a "pump" drift wave given by

$$
\xi_{0}=\sum_{m} \xi_{m}^{0}(x) e^{-i \omega_{0} t+i n \zeta+i m \theta}
$$

Here $\xi$ represents the density, scalar potential perturbations associated with the pump drift wave. The summation over $m$ is for a few symmetric sidebands around the primary mode number $m_{0}=n / q$, where $q$ is the safety factor at the rational surface. This mode can couple to the GAM mode, which is represented as

$$
\xi_{G}=\left[\phi_{G}(x), n_{G}(x, \theta)\right] e^{-i \omega t} .
$$

For the GAM the potential is independent of the variable $\theta$, while the density has a $\sin \theta$ dependence. Here the radial wavenumber dependence is not written explicitly as a wavenumber as done in earlier work for the homogeneous case. ${ }^{18}$ It nevertheless appears through the spatial derivative in $x$. The inhomogeneity effectively makes the radial wavenumber nonlocal.

These two modes, namely the pump drift wave and the GAM, excite a resonant sideband,

$$
\xi_{s}=\sum_{m} \xi_{m}^{s}(x) e^{-i\left(\omega-\omega_{0}\right) t-i n \zeta-i m \theta} .
$$

Using these representations, the equations for the driven GAM and sideband modes (retaining only the dominant nonlinear coupling) can be written as ${ }^{18}$

$$
\begin{aligned}
& {\left[\omega^{2}-\omega_{G}^{2}(x)\right] \psi_{G}=-k_{y} \rho_{s 0} c_{s 0} \omega_{G}(0) \phi_{0} \frac{d^{2} \phi_{s}}{d x^{2}}+\frac{D}{n_{0}}} \\
& \left(\omega_{0}-\omega\right)\left[\left(1+k_{y}^{2} \rho_{s 0}^{2}\right) \phi_{s}-\frac{\rho_{s 0}^{2}}{n_{0}} \frac{d}{d x}\left(n_{0} \frac{d}{d x} \phi_{s}\right)\right] \\
& \quad+k_{y} \rho_{s 0} c_{s 0}\left(\frac{1}{n_{0}} \frac{d n_{0}}{d x}\right) \phi_{s} \\
& \quad=k_{y} \rho_{s 0} c_{s 0}\left[\phi_{0}^{*}\left(1+k_{y}^{2} \rho_{s 0}^{2}\right) \psi_{G}+\frac{\rho_{s 0}^{2}}{n_{0}} \frac{d}{d x}\left(n_{0} \phi_{0}^{*} \frac{d}{d x} \psi_{G}\right)\right],
\end{aligned}
$$

where $\psi_{G}=d \phi_{G} / d x, \omega_{G}(x)=\sqrt{2} c_{s}(x) / R, k_{y}=m_{0} / a$, where $a$ is the minor radius of the plasma. $D$ is a constant of integration.
As shown in earlier work $^{12}$ the dominant nonlinear coupling is to the primary mode $m_{0}=n / q$, where $q$ is the safety factor at the rational surface. There are couplings to the $m_{0} \pm 1$ sidebands which turn out to be $1 / m_{0}$ smaller than the dominant terms.

For a homogeneous plasma, $n_{0}=1, T_{e}=1$, and assuming that both $\psi_{G}(x)=\hat{\psi}_{G} e^{i q_{r} x}$ and $\phi_{s}(x)=\hat{\phi}_{s} e^{i q_{r} x}$, the original algebraic coupled equations [Eqs. (10) and (11) of Ref. 18] derived earlier are recovered.

In the absence of the coupling to the sideband drift wave $\phi_{s}$, the second term on the right hand side of Eq. (6) is what gives rise to the continuum GAMs. There are various suggestions as to how to create discrete eigenmodes by the inclusion of both finite Larmor radius effects and finite orbit effects. Here we show that in the presence of coupling to drift waves, there are particular solutions $(D=0)$ which can be constructed that are indeed discrete "global" eigenmodes localized in the region of the density and temperature pedestals.

\section{DIMENSIONLESS EQUATIONS}

Equations (6) and (7) are studied numerically for tokamak edge-like profiles. The equations are first rendered dimensionless and this yields four dimensionless parameters. In the edge region the normalized density profile is represented by

$$
n_{0}(x)=1-\Delta_{n} \tanh \left(\frac{x}{L_{n} \Delta_{n}}\right),
$$

with

$$
\Delta_{n}=\frac{n_{1}-n_{2}}{n_{1}+n_{2}} .
$$

Here $n_{1}$ and $n_{2}$ can be adjusted to determine the height of the pedestals and $L_{n}$ determines the characteristic scale-length of the pedestal at $x=0$. A similar expression is used for the electron temperature profile. In the present studies the ratio of the density and temperature gradient scale-lengths has been assumed to be unity. Thus with this choice of the profile a convenient normalization scale-length in $x$ will be $L_{n}$. The frequency is normalized to the GAM frequency at $x=0$.

The dimensionless equations are

$$
\begin{aligned}
& \left(\Omega_{0}-\Omega\right)\left[\left(1+k_{y}^{2} \rho_{s 0}^{2}\right) \phi_{s}-\frac{\rho_{s 0}^{2}}{L_{n}^{2} n_{0}} \frac{d}{d x}\left(n_{0} \frac{d}{d x} \phi_{s}\right)\right]-\Omega_{*}(x) \phi_{s} \\
& =\left[\Gamma\left(1+k_{y}^{2} \rho_{s 0}^{2}\right) \psi_{G}+\frac{\rho_{s 0}^{2}}{L_{n}^{2} n_{0}} \frac{d}{d x}\left(n \Gamma \frac{d}{d x} \psi_{G}\right)\right] \\
& {\left[\Omega+\Omega_{G}(x)\right] \psi_{G}=\chi_{G},} \\
& {\left[\Omega-\Omega_{G}(x)\right] \chi_{G}=-\Gamma \frac{d^{2} \phi_{s}}{d x^{2}} .}
\end{aligned}
$$

Here $\Omega=\omega R / \sqrt{2} c_{s 0}, \quad \Omega_{0}=k_{y} \rho_{s 0} R / \sqrt{2} L_{n}\left(1+k_{y}^{2} \rho_{s 0}^{2}\right)$, and $\Gamma$ $=\left(k_{y} \rho_{s 0} R / \sqrt{2} L_{n}\right)\left|\phi_{0}\right|$. The normalized GAM frequency is just the square root of the normalized temperature which has a functional form similar to that of the density profile given 

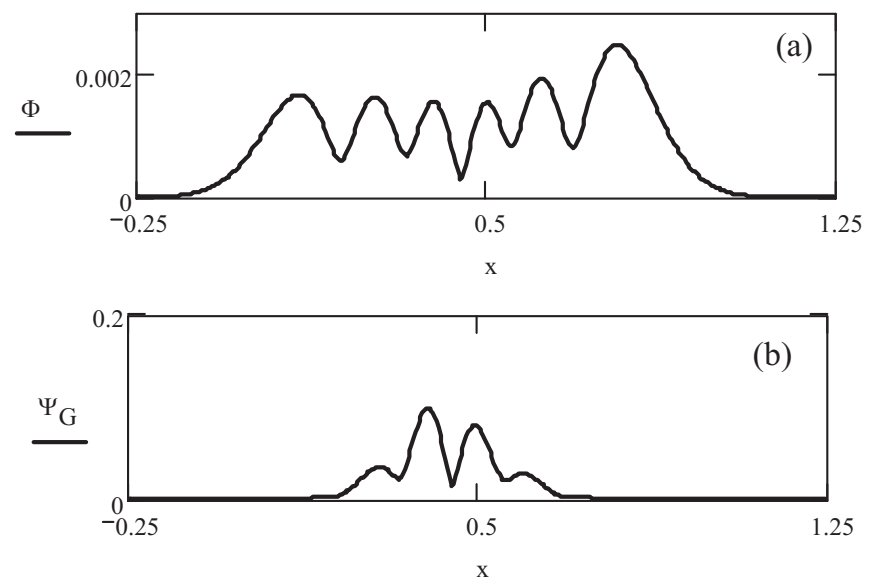

FIG. 1. (a) $\Phi=\left|\phi_{s}\right|$ and (b) $\Psi_{G}=\left|\psi_{G}\right|$ as a function of $x$ for $k_{y} \rho_{s 0}=0.5, \varepsilon_{n}$ $=0.1, \rho_{s 0} / L_{n}=0.02$, and $\Phi_{0}=2 \times 10^{-3}$.

above. The equation for the GAM [Eq. (6)] has been split into the two equations. This is done so as to allow the equations to be cast into the form of a traditional matrix eigenvalue problem once the differential operators are finite differenced. Finally there are four dimensionless parameters, (1) $k_{y} \rho_{s 0}$, (2) $\rho_{s 0} / L_{n}$, (3) $\varepsilon_{n}=\sqrt{ } 2 L_{n} / R$, and (4) $\Phi_{0}=e \phi_{0} / T_{e}$.

There is an additional equation that first needs to be solved to determine the eigenvalue and spatial structure of the pump wave in the prescribed density profile. This is

$$
\Omega_{0}\left[\left(1+k_{y}^{2} \rho_{s 0}^{2}\right) \phi_{0}-\frac{\rho_{s 0}^{2}}{L_{n}^{2} n_{0}} \frac{d}{d x}\left(n_{0} \frac{d}{d x} \phi_{0}\right)\right]-\Omega_{*}(x) \phi_{0}=0
$$

The lowest radial harmonic $\left(n_{r}=0\right)$ is chosen as the pump wave.

\section{NUMERICAL RESULTS}

For $k_{y} \rho_{s 0}=0.4, \varepsilon_{n}=0.1, \rho_{s 0} / L_{n}=0.02$ and $\Phi_{0}=2 \times 10^{-3}$, shown in Figs. 1(a) and 1(b) are the modulus of the eigenfunctions of the sideband drift wave and GAM modes, respectively, for the most unstable mode. There are various interesting features worth noting. The first obvious feature is the two scale character of the eigenfunctions. As noted in earlier studies the excitation of the GAM is caused by a resonant parametric process. Thus at some specific point on the density/temperature profile the resonance condition is satisfied. The resonance condition yields ${ }^{18}$ the following relationship for the local radial wavenumber $q_{r}$ :

$$
\frac{\omega_{*} q_{r}^{2} \rho_{s}^{2}\left(x_{0}\right)}{\left[1+k_{y}^{2} \rho_{s}^{2}\left(x_{0}\right)\right]\left[1+\left(q_{r}^{2}+k_{y}^{2}\right) \rho_{s}^{2}\left(x_{0}\right)\right]}=\frac{\sqrt{2} c_{s}\left(x_{0}\right)}{R},
$$

where $x_{0}$ is the location where the resonance occurs. The wavenumber $q_{r}$ is responsible for the "fast" scale seen in the wavefunctions shown in Fig. 1. For the nonlocal problem, the radial eigenmodes are characterized by discrete radial "quantum" numbers " $n_{r}$." For the present case, the discrete mode number is $n_{r}=5$. Due to the plasma density and temperature inhomogeneity, the resonance gets detuned and the local parametric growth is detuned. Hence, the three-wave

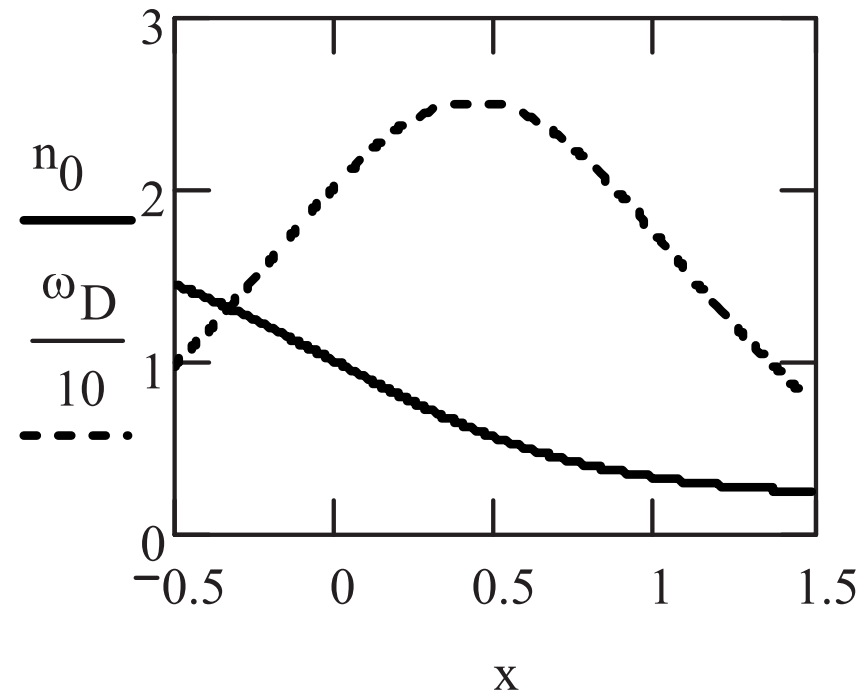

FIG. 2. The density and diamagnetic frequency (/10) as a function of $x$ in the region of the mode localization. The parameters are the same as those used in Fig. 1.

interaction process is localized on the "slow" scale $\sim O\left[\left(\rho_{s 0} / L_{n}\right)^{1 / 2}\right]$ (in these normalized units) determined by the inhomogeneity scales present in the ambient plasma.

The second interesting feature is the drift wave sideband eigenfunction [Fig. 1(a)] is not centered at the location of the steepest density and temperature gradients $(x=0)$ but at $x$ $=x_{0}=0.44$.

What determines this location? In Fig. 2 is plotted the normalized density profile and the normalized drift frequency (reduced by a factor of 10). What is evident is that the diamagnetic frequency is a maximum at $x=0.44$ and this determines the minimum of the "effective" potential well for localization of the global drift wave mode. The location of the global GAM mode [Fig. 1(b)] excited by drift waves is however localized is the region where the drive mechanism best satisfies the resonance condition. Thus the peak of the eigenfunction [Fig. 1(b)] is at the resonance point. The frequency and growth rate for the GAM in normalized units are $\Omega_{r}=0.812$ and $\Omega_{l}=0.064$, respectively. The characteristic fast scale-lengths for the two modes are the same as required by the resonance condition. The slow scales are however different. For the sideband drift wave the characteristic "slow" scale $\sim \sqrt{n_{r}}\left[\rho_{s 0} / L_{n}\left(x_{0}\right)\right]^{1 / 2}$, where $n_{r}$ is the radial quantum number. However for the GAM, the width is determined by the pump width.

Shown in Fig. 3 is the spectrum of modes in the frequency $\left(\omega_{r}\right)$ growth rate $\left(\omega_{i}\right)$ space. There are primarily two complex conjugate pairs as well as a band of continuum modes. The densely packed continuum modes arise from the very high spatial resolution (400 grid points) used in these computations.

The second unstable mode (the left one in the diagram) has a frequency and growth rate given by $\Omega_{r}=0.742$ and $\Omega_{l}=0.047$. What is interesting to see is that the eigenfunction (Fig. 4) for this second unstable mode has a radial quantum number $n_{r}=4$ and the peak of the eigenfunction for the GAM 


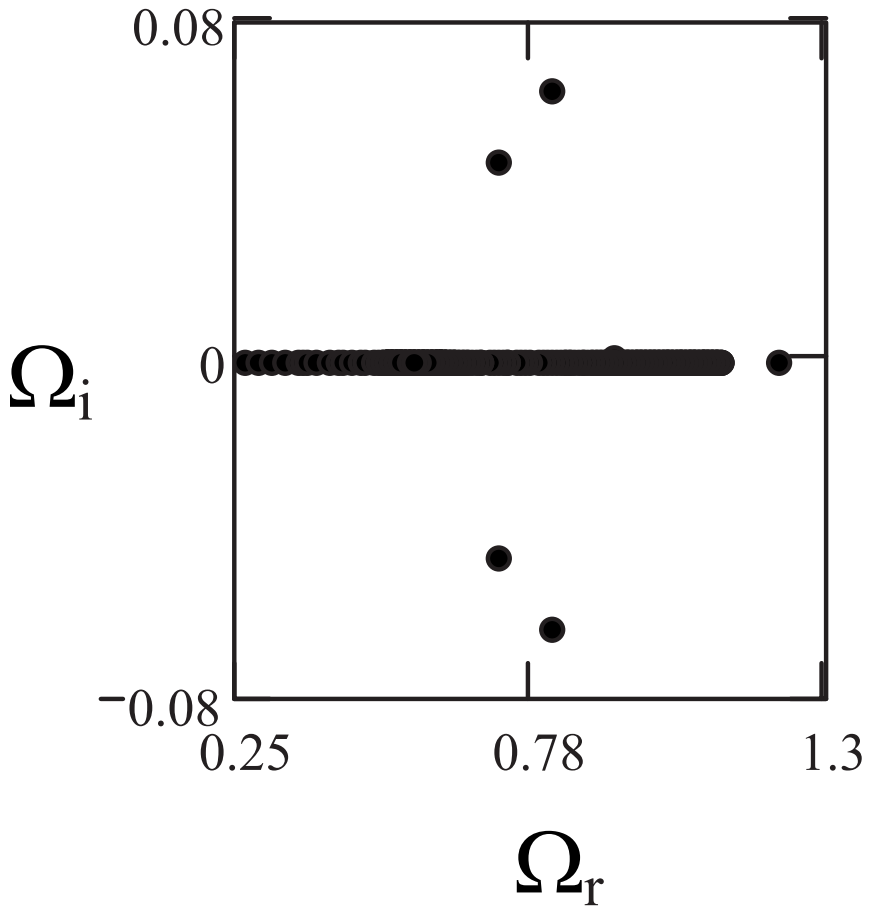

FIG. 3. Real vs imaginary frequencies $\Omega_{r}$ and $\Omega_{i}$. The parameters are the same as those used in Fig. 1.

is at a different resonant location to the right of the most unstable mode.

This characteristic two scale feature is seen over a very broad range of parameters, the separation between the "fast" scale and "slow" scale becoming more and more disparate with the decrease in $\rho_{s 0} / L_{n}$. This two scale eigenmodes feature can qualitatively account for the very large spread in the values of the measured radial wavenumbers. Those studies which use reflectrometry techniques would invariably measure the "fast" scale, while the multipin probes or reciprocating probes would mostly sample the "slow" scale. The "fast" scales would yield $q_{r} \rho_{s 0} \sim O\left(10^{-1}-1\right)$, while the "slow" scales can account for $q_{r} \rho_{s 0} \sim O\left(10^{-2}-10^{-1}\right)$.

The question that comes to mind is why are there only two strongly unstable modes? The answer to this question is
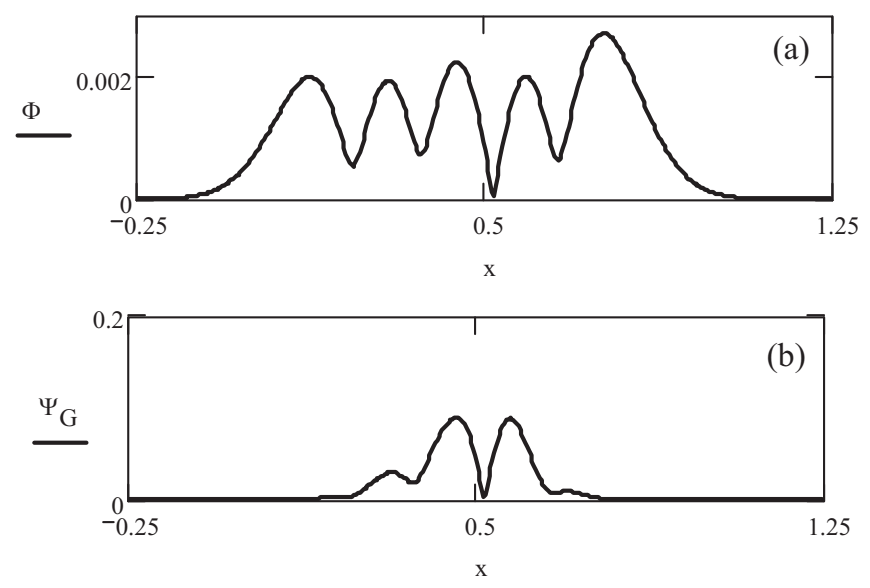

FIG. 4. (a) $\Phi=\left|\phi_{s}\right|$ and (b) $\Psi_{G}=\left|\psi_{G}\right|$ as a function of $x$ for the second unstable mode. The parameters are the same as those used in Fig. 1.

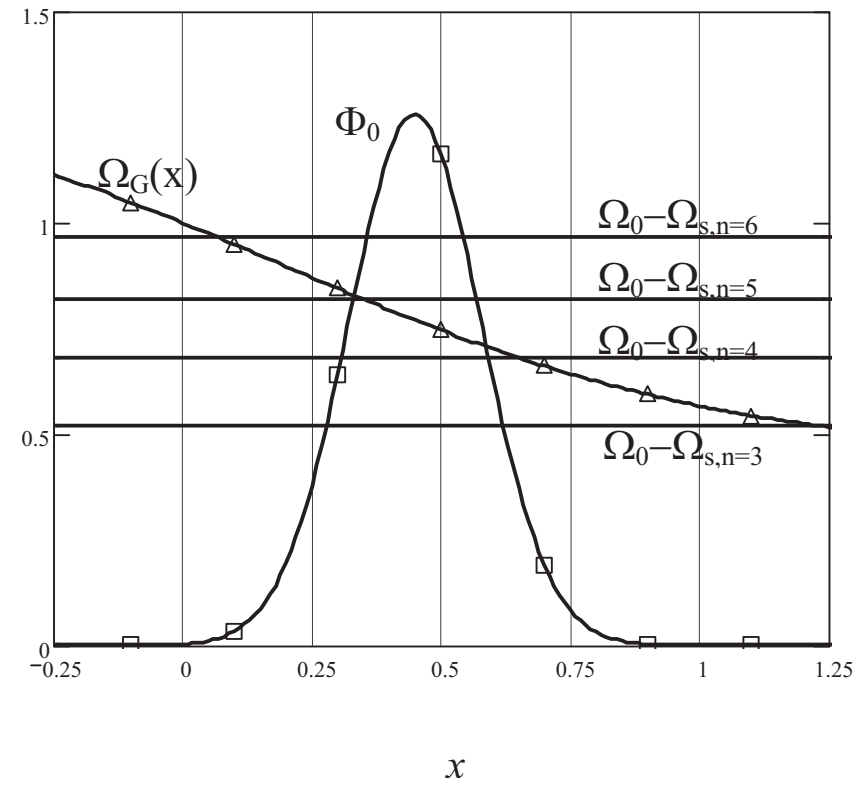

FIG. 5. GAM frequency $\Omega_{G}(x)$ (triangles), pump amplitude $\Phi_{0}$ (squares), and difference frequencies $\Omega_{0}-\Omega_{s}$ for radial mode numbers $n_{r}=3,4,5,6$ for the drift wave sidebands (labeled solid curves) as a function of $x$. The parameters are the same as those used in Fig. 1.

addressed in Fig. 5. Shown in this plot is the variation of the GAM frequency $\Omega_{G}(x)$ as a function of $x$ (solid curve with triangles), the spatial structure of the drift wave pump wave $\Phi_{0}$ (solid curve with squares) and the difference frequency (between the pump drift wave and the sideband drift waves) $\Omega_{0}-\Omega_{s}$ for radial mode numbers $n_{r}=3,4,5$, and 6 (solid lines) for the sideband. The location of the three-wave resonances is at the intersection points between the GAM frequency curve and the four difference frequencies. At the location of the resonances with the $n_{r}=4,5$ modes, the pump wave amplitude is of significant magnitude, while at the location of the resonances with the $n_{r}=3$ and $n_{r}=6$ modes, the amplitude has dropped off significantly due to the radial localization of the pump wave. This explains why there are dominantly two strongly unstable modes for this choice of parameters. Furthermore, Fig. 3 indicates that the $n_{r}=5$ resonance is more strongly growing compared to the $n_{r}=4$ case. This is because the amplitude of the pump (as shown in Fig. 5 ) is larger at the $n_{r}=5$ resonance compared to its magnitude at the $n_{r}=4$ resonance point.

Next, the variation of the growth rate as a function of $k_{y} \rho_{s 0}$ is computed and compared with that given by the local theory. In these normalized units, the local theory yields the following expressions for the resonant radial wavenumber and the growth rate:

$$
\begin{gathered}
\frac{q_{r 0} \rho_{s 0}}{L_{n}}=\left[\frac{\Omega_{*}\left(x_{0}\right)}{\Omega_{0}-\Omega_{G}\left(x_{0}\right)}-1-k_{y}^{2} \rho_{s 0}^{2}\right]^{1 / 2}, \\
\gamma=k_{y} q_{r 0} \rho_{s 0}^{2}\left[\frac{1+k_{y}^{2} \rho_{s 0}^{2}-\frac{q_{r 0}^{2} \rho_{s 0}^{2}}{L_{n}^{2}}}{2 \Omega_{G}\left(x_{0}\right)\left(1+k_{y}^{2} \rho_{s 0}^{2}+\frac{q_{r 0}^{2} \rho_{s 0}^{2}}{L_{n}^{2}}\right)}\right]^{1 / 2} .
\end{gathered}
$$

The only difference in this local dispersion relationship compared to that derived in earlier work is that the drift fre- 

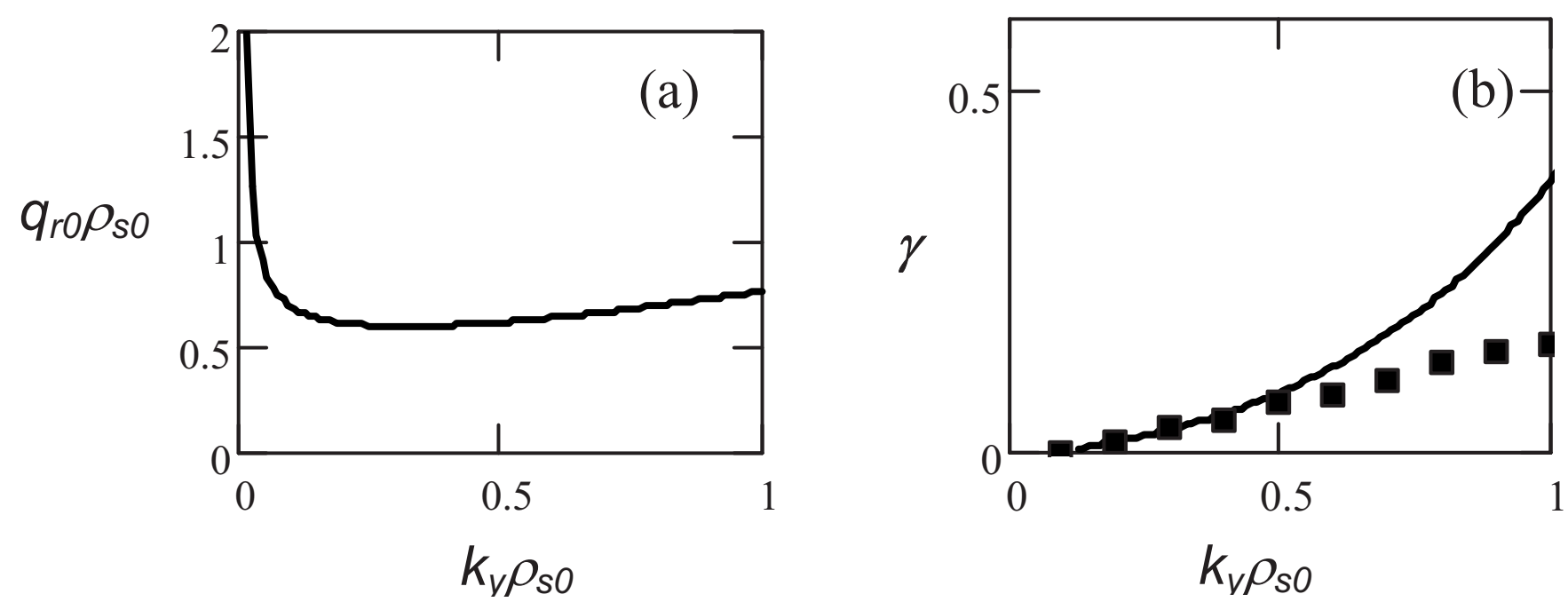

FIG. 6. (a) $q_{r 0} \rho_{s 0}$ vs $k_{y} \rho_{s 0}$ for $\varepsilon_{n}=0.1, \rho_{s 0} / L_{n}=0.02$, and $\Phi=2 \times 10^{-3}$. (b) Growth rate $\gamma$ vs $k_{y} \rho_{s 0}$ from local theory (solid line) [Eq. (11)] and from nonlocal equations (maximum value) (solid squares) [Eqs. (8) and (9)].

quency and the GAM frequency are evaluated at the position of the local maxima of the drift frequency at $x=x_{0}$, since the earlier nonlocal analysis indicates that the modes are localized around this point. Shown in Fig. 6(a) is the resonant wavenumber $q_{r 0} \rho_{s 0}$ as a function of $k_{y} \rho_{s 0}$.

Thus, as expected the resonance condition for the given choice of parameters gives the "fast" scale for which $q_{r} \rho_{s 0}$ $\sim O(0.6-1)$ for the unstable modes. In Fig. 6(b) the nonlocal growth rate of the fastest growing mode (solid squares) are compared with those obtained from the local theory evaluated at the position of the maximum of the diamagnetic frequency. The GAM frequency is also computed at that location. It is clear that the local theory does not agree quantitatively with the growth rates computed using the nonlocal equations when $k_{y} \rho_{s 0}>0.5$ but has the same trend as a function of $k_{y} \rho_{s 0}$.

Shown in Fig. 7 is the spread in the real frequency of the excited GAMs. In Fig. 7(a) is show the GAM frequency of the maximally growing mode as a function of $k_{y} \rho_{s 0}$ for $\varepsilon_{n}$ $=0.1, \rho_{s 0} / L_{n}=0.02$ and $\Phi_{0}=2 \times 10^{-3}$. The spread in the frequency is about $20 \%$. Displayed in Fig. 7(b) is the frequency $\left(\Omega_{r}\right)$ and imaginary frequency $\left(\Omega_{i}\right)$ of the GAMs for $\varepsilon_{n}$ $=0.1, \rho_{s 0} / L_{n}=0.02, k_{y} \rho_{s 0}=0.8$ and $\Phi_{0}=2 \times 10^{-3}$. For the unstable GAMs the real frequency spread is about $20 \%$. It has to be remembered that the amplitude of the drift wave which determines the growth as well as the real frequency has been fixed at $\Phi_{0}=2 \times 10^{-3}$ for the data presented in Fig. 6. Higher amplitudes lead to an increase in the growth rate of the unstable modes. However the width of the real frequency spectrum over which the modes are unstable does not increase significantly but the density of unstable modes within this width does.

This may account for the observed feature in the bispectrum analysis ${ }^{15,16}$ performed on various devices which indicate that a very large spread in the "high" frequency modes (represented by the large spread in $k_{y} \rho_{s}$ ) couples (three-wave process) to a very narrow spectrum (near "monochromatic") in frequency of the GAMs.

Figure 7(b) clearly shows that for certain parameters
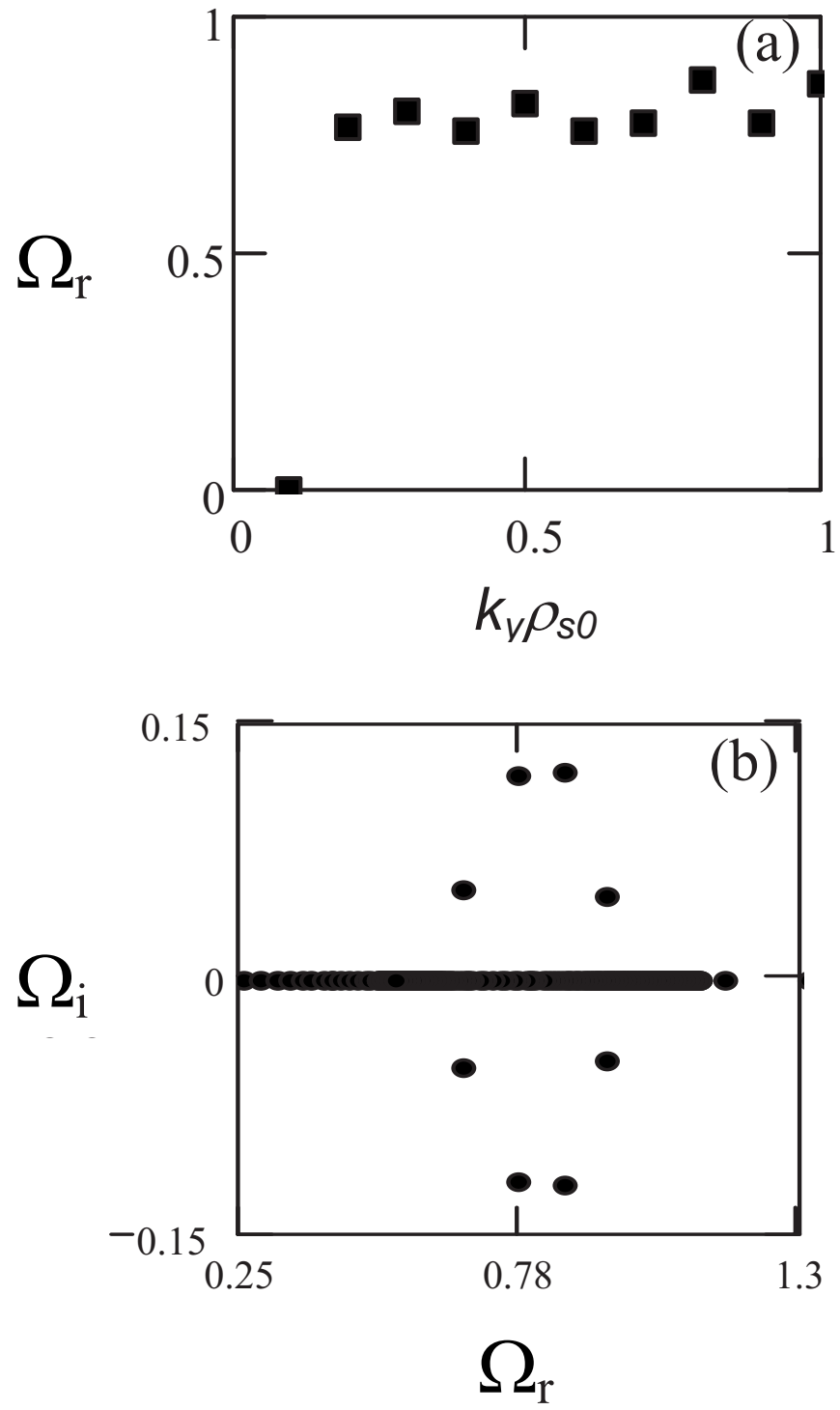

FIG. 7. (a) GAM frequency $\left(\omega_{r}\right)$ of the maximally growing mode as a function of $k_{y} \rho_{s 0}$, for $\varepsilon_{n}=0.1, \rho_{s 0} / L_{n}=0.02$ and $\Phi_{0}=2 \times 10^{-3}$. (b) Real frequency $\left(\Omega_{r}\right)$ and imaginary frequency $\left(\Omega_{i}\right)$ of the GAMs for $\varepsilon_{n}=0.1$, $\rho_{s 0} / L_{n}=0.02, k_{y} \rho_{s 0}=0.8$ and $\Phi_{0}=2 \times 10^{-3}$. 


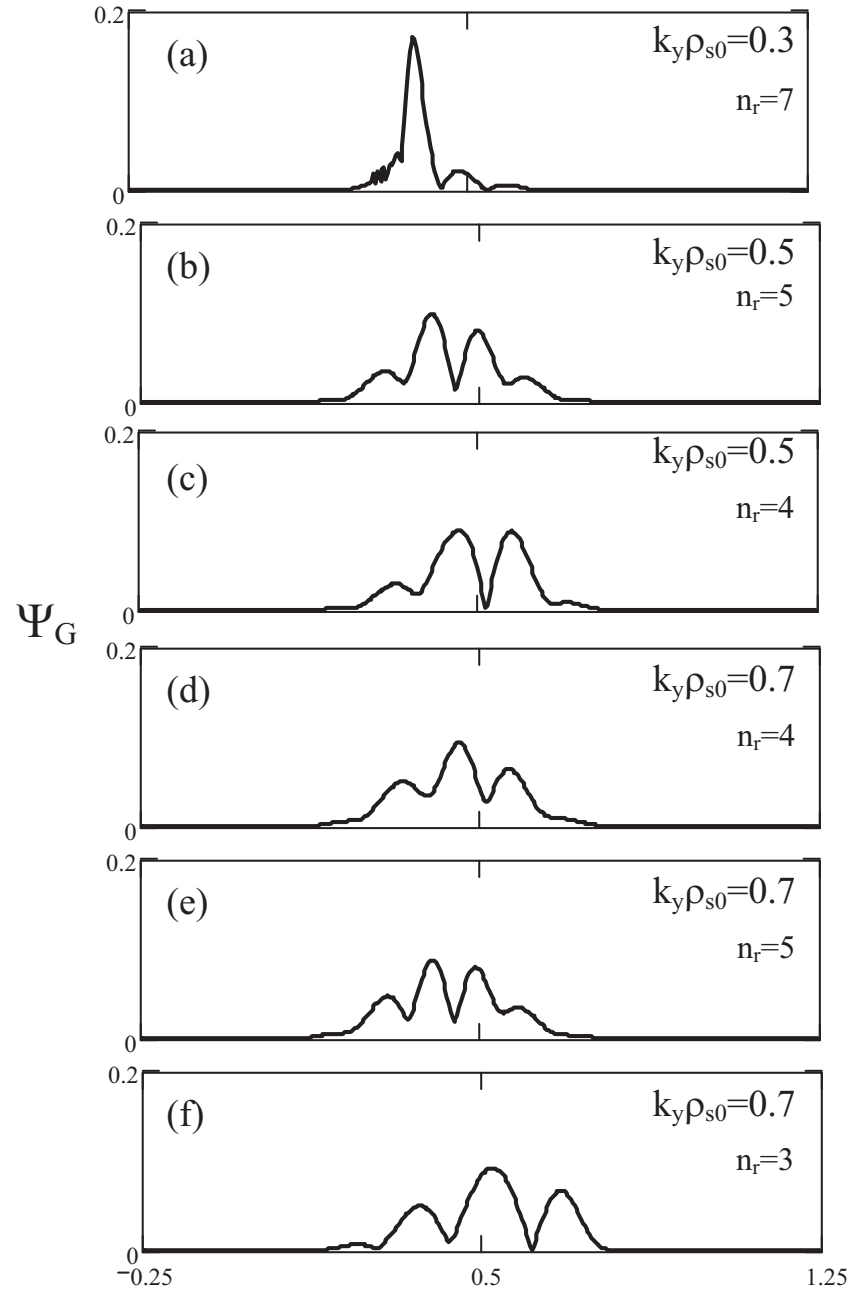

FIG. 8. Eigenfunction $\Psi_{G}$ of the six unstable GAMs for $\varepsilon_{n}=0.1, \rho_{s 0} / L_{n}$ $=0.02$, and $\Phi_{0}=2 \times 10^{-3}$, (a) $k_{y} \rho_{s 0}=0.3, n=7$, (b) $k_{y} \rho_{s 0}=0.5, n_{r}=5$, (c) $k_{y} \rho_{s 0}=0.5, n_{r}=4$ (d) $k_{y} \rho_{s 0}=0.7, n_{r}=4$, (e) $k_{y} \rho_{s 0}=0.7, n_{r}=5$, and (f) $k_{y} \rho_{s 0}$ $=0.7, n_{r}=3$.

there can be multiple unstable eigenmodes. The spatial structure of these modes is the focus of the next figure. Shown in Fig. 8 is the modulus of the eigenfunction of the GAM $\Psi_{G}$ for six unstable modes for different values of $k_{y} \rho_{s 0}$ and radial "quantum" number $n_{r}$.

There are many interesting aspects to this plot. The eigenfrequency increases as the peak of the eigenmode shifts towards spatially increasing GAM frequency. The discrete eigenfrequencies are determined by the resonance between the spatially varying GAM frequency and the drift wave sideband eigenmodes. For these modes the radial mode number $n_{r}$ varies from 3 to 7 . There is significant spatial overlap in the eigenmodes of some of the modes.

Finally, in Fig. 9 is shown the spatial variation of the normalized GAM frequency and the peak of the location of the fastest growing eigenfunctions (open squares) and second fastest growing modes (open triangles) for $k_{y} \rho_{s 0}$ $=0.2,0,3,0.4,0.5,0.6,0.7,0.8,0.9,1.0$. There is only one unstable radial mode for $k_{y} \rho_{s 0}=0.4,0.7$. The parameters used for this plot are $\varepsilon_{n}=0.1, \rho_{s 0} / L_{n}=0.02$, and $\Phi_{0}=5 \times 10^{-4}$. There are sixteen unstable modes displayed in this plot. Clearly, there is significant spatial overlap between some of

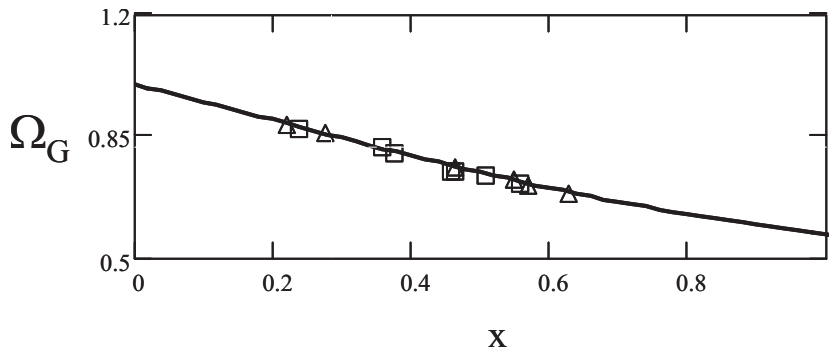

FIG. 9. The solid curve is the normalized GAM frequency as a function of $x$. Open squares are the location of the peaks of the fastest growing eigenmodes for $k_{y} \rho_{s 0}=0.2,0,3,0.4,0.5,0.6,0.7,0.8,0.9,1.0$ and the open triangles are for the second fastest growing modes for $k_{y} \rho_{s 0}$ $=0.2,0,3,0.5,0.6,0.8,0.9,1.0$. The parameters $\varepsilon_{n}=0.1, \rho_{s 0} / L_{n}=0.02$ and $\Phi=5 \times 10^{-4}$.

the modes. Thus it is a combination of variation in $k_{y} \rho_{s 0}$ and the radial mode number which determines the density and spatial distribution of the resonances and hence the location of multiple eigenmodes. Hence the spatial structure is very intimately tied to the excitation mechanism of the GAMs, namely the drift waves. This current model qualitatively displays the spatial features of the multiple eigenmodes observed by the Doppler reflectometry measurements presented in the work of Conway et al. ${ }^{14}$ with the difference that the frequencies are near the local GAM frequency rather than at some scaled GAM frequency. For the present study the growth rates are a very small fraction of the GAM frequency. Thus the resonances are dominantly the linear resonances.

\section{CONCLUSIONS}

In this paper calculations are presented which address the issue of excitation of the geodesic acoustic mode by nonlinear mode coupling to drift waves in an inhomogeneous plasma with density and electron temperature gradients. It is shown that the traditional GAM which is a continuum mode can be converted into global eigenmodes due to the parametric three-wave coupling to drift waves. The present study shows that this three-wave resonant process in the presence of inhomogeneity gives rise to a two scale structure of the global unstable eigenmodes. The "fast" radial scale is determined by the wave-frequency matching conditions, while the "slow" scale is determined by the inhomogeneity scale. Thus the different experimental techniques used for studying the structure of the GAM will depend on whether they sample the "fast" scale or the "slow" scale. Reflectometry techniques which rely on Bragg scattering conditions will give measurements of the "fast" scale for which $q_{r} \rho_{s 0} \sim O\left(10^{-1}-1\right)$. Multipin probes or reciprocating probes would on the other hand give measurements of the "slow" scale with $q_{r} \rho_{s 0}$ $\sim O\left(10^{-2}-10^{-1}\right)$. The excitation mechanism basically determines the localization of the GAMs and since drift waves are the proposed natural "pump" waves the modes are mostly localized in the edge region of these devices. Thus it is probably a combination of the mechanism and the preferential damping of GAMs in the core (due to stronger Landau damping) that makes GAMs an edge phenomenon. Finally even if there is a very broad spectrum of drift waves, $(0.1$ $\left.<k_{y} \rho_{s 0} \sim 1\right)$ they can excite GAMs with frequencies which 
are almost "monochromatic," since the GAM frequency has a spread of at most $20 \%$ over the radial extent of the drift waves which are localized by the variation in the density in the edge region of tokamaks. This may explain the structure of the bicoherence spectra ${ }^{15,16}$ observed on a variety of devices. Finally, the multiple discrete eigenmodes observed in the edge pedestal region ${ }^{14}$ can be the interpreted as nonlocal eigenmodes driven by a spectrum of drift waves.

\section{ACKNOWLEDGMENTS}

The work of P. N. Guzdar and R. G. Kleva was supported by a grant from the U.S. Department of Energy. Also V. Naulin and J. J. Rasmussen were supported by the Danish Natural Science Research Council (Grant No. FNU-272-060367).

${ }^{1}$ G. R. McKee, R. J. Fonck, M. Jakubowski, K. H. Burrell, K. Hallatschek, R. A. Moyer, D. L. Rudakov, W. Nevins, G. D. Porter, P. Schoch, and X. Xu, Phys. Plasmas 10, 1712 (2003).

${ }^{2}$ H. Punzmann and M. G. Shats, Phys. Rev. Lett. 93, 125003 (2004).

${ }^{3}$ G. Conway, B. Scott, J. Schirmer, M. Reich, A. Kendl, and the ASDEX Upgrade Team, Plasma Phys. Controlled Fusion 47, 1165 (2005).

${ }^{4}$ T. Ido, Y. Muira, K. Kamiya, Y. Hamada, K. Hoshino, A. Fujisawa, K. Itoh, S-I Itoh, A. Nishizawa, H. Ogawa, Y. Kusama, and JFT-2M Group, Plasma Phys. Controlled Fusion 48, S41 (2006).

${ }^{5}$ G. R. McKee, D. K. Gupta, R. J. Fonck, D. J. Schlossberg, M. W. Shafer, and P. Gohil, Plasma Phys. Controlled Fusion 48, S123 (2006).

${ }^{6}$ A. V. Melnikov, V. A. Vershkov, L. G. Eliseev, S. A. Grashin, A. V. Gudoznik, L. I. Krupnik, S. E. Lysenko, V. A. Mavrin, S. V. Perfilov, D. A. Shelukin, S. V. Soldatov, M. V. Ufimtsev, A. O. Urazbaev, G. Van
Oost, and L. G. Zimeleva, Plasma Phys. Controlled Fusion 48, S87 (2006).

${ }^{7}$ K. J. Zhao, T. Lan, J. Q. Dong, L. W. Lan, W. Y. Hong, C. X. Yu, A. D. Liu, J. Qian, J. Cheng, D. L. Yu, Q. W. Yang, X. T. Ding, Y. Liu, and C. H. Pan, Phys. Rev. Lett. 96, 255004 (2006).

${ }^{8}$ N. Winsor, J. L. Johnson, and J. M. Dawson, Phys. Fluids 11, 2448 (1968).

${ }^{9}$ B. D. Scott, Plasma Phys. Controlled Fusion 39, 1635 (1997).

${ }^{10}$ K. Hallatschek and D. Biskamp, Phys. Rev. Lett. 86, 1223 (2001).

${ }^{11}$ V. Naulin, Phys. Plasmas 10, 4016 (2003).

${ }^{12}$ B. D. Scott, N. J. Phys. 7, 92 (2005).

${ }^{13}$ V. Naulin, A. Kendl, O. E. Garcia, A. H. Nielsen, and J. J. Rasmussen, Phys. Plasmas 12, 052515 (2005).

${ }^{14}$ G. D. Conway and the ASDEX Upgrade Team, Plasma Phys. Controlled Fusion 50, 085005 (2008).

${ }^{15}$ A. Fujisawa, T. Ido, A. Shimizu, S. Okamura, K. Matsuoka, H. Ihuchi, Y. Hamada, H. Nakano, S. Oshima, K. Itoh, K. Hoshino, K. Shinohara, Y. Muira, Y. Nagashima, S.-I. Itoh, M. Shats, H. Xia, J. Q. Dong, L. W. Yan, K. J. Zhao, G. D. Conway, U. Stroth, A. V. Melnikov, L. G. Eliseev, S. E. Lysenko, S. V. Perfolov, C. Hidalgo, G. R. Tynan, C. Holland, P. H. Diamond, G. R. McKee, R. J. Fonck, D. K. Gupta, and P. M. Schoch, Nucl. Fusion 47, S718 (2007).

${ }^{16}$ T. Lan, A. D. Liu, C. X. Yu, L. W. Lan, W. Y. Hong, K. J. Zhao, J. Q. Dong, J. Qian, J. Cheng, D. L. Lu, and Q. W. Yang, Plasma Phys. Controlled Fusion 50, 045002 (2008).

${ }^{17}$ K. Itoh, K. Hallatschek, and S. Itoh, Plasma Phys. Controlled Fusion 47, 451 (2005).

${ }^{18}$ N. Chakrabarti, R. Singh, P. K. Kaw, and P. N. Guzdar, Phys. Plasmas 14, 052308 (2007).

${ }^{19}$ F. Zonca and L. Chen, Europhys. Lett. 83, 35001 (2008).

${ }^{20}$ P. N. Guzdar, N. Chakrabarti, R. Singh, and P. K. Kaw, Plasma Phys. Controlled Fusion 50, 025006 (2008).

${ }^{21}$ N. Chakrabarti, P. N. Guzdar, R. G. Kleva, V. Naulin, J. J. Rasmussen, and P. K. Kaw, Phys. Plasmas 15, 112310 (2008).

${ }^{22}$ A. Zeiler, J. F. Drake, and B. Rogers, Phys. Plasmas 4, 2134 (1997). 